\title{
Responses to the Challenges of Fast Digital Conversion, in the Light of International Research Results - A Comparative Look at Virtual Spaces
}

\author{
Nagy Katalin', Orosz Beáta², Szüts Zoltán ${ }^{3}$, Balogh Zoltán ${ }^{4}$, \\ Martin Magdin ${ }^{4}$, Stefan Koprda ${ }^{4}$, Pintér Róbert ${ }^{5}$, \\ Molnár György ${ }^{1}$
}

${ }^{1}$ Department of Technical Education, Budapest University of Technology and Economics, Müegyetem rkp. 3, 1111 Budapest, Hungary; nagy.katalin@gtk.bme.hu, molnar.gyorgy@gtk.bme.hu

${ }^{2} \mathrm{PhD}$ School in Business and Management, Budapest University of Technology and Economics, Müegyetem rkp. 3, 1111 Budapest, Hungary, e-mail:

orosz@metakepzes.hu

${ }^{3}$ Institute of Digital Technology, Eszterházy Károly University, Eszterházy tér 1, 3300 Eger, Hungary; szuts.zoltan@uni-eszterhazy.hu

${ }^{4}$ Department of Informatics, Faculty of Natural Sciences, Constantine the Philosopher University in Nitra, Tr. A. Hlinku 1, 94974 Nitra, Slovakia; zbalogh@ukf.sk,mmagdin@ukf.sk and skoprda@ukf.sk

${ }^{5}$ Department of Informatics, Subotica Tech, Subotica, Serbia, pinter.robert@vts.su.ac.rs

\footnotetext{
Abstract: In order to assure effectiveness, digital instruction prioritizes digital devices and systems over traditional technological and methodological solutions. In the current digital age and the way of life defined by digital culture, digital skills and competences become highly appreciated. Also, innovative methodological solutions, promoting the long term maintenance of attention and motivation, become key factors in remote learning or digitally scheduled education. Furthermore, the emergence of additional digital gaps, triggers newer digital paradigm shifts, gaining special importance during such exigencies, as a pandemic. An effective response or potential remedy, to this situation, is offered by digital pedagogy. Digital pedagogy faced substantial challenges during the first wave of the COVID-19 pandemic, imposing demanding tasks on all participants, within the education sector. Our study introduces the results of a quantitative, multivariable, empirical inquiry, entailing a comparative analysis of data obtained from a survey of the pedagogue attitudes and digital tool systems for three selected countries.
} 
Keywords: COVID-19; Virtual Learning Environments; digital tools and systems; digital competences

\section{Introduction}

Several factors including the availability of the trained labour force or the adaptation skills of the members of society can determine the economic development of a country. Identifying a correlation between the available human capital and the quality of education reveals that quality improvement can lead to better labour market perspectives. [1-7]. Hanushek and Kimko [5] were able to quantify quality in the context of internationally comparable test results. Consequently, while a quality increase equal to one unit of standard deviation results in a growth rate of 1 percentage point, the achievement of the same result requires nine years of schooling on average if the given intervention is only quantity-oriented [4]. The efficiency of a country's education system and its growth potential are determined by the increasing personal knowledge of teachers as teacher quality represents a variable with a direct impact on students' academic progress. Paper [3] asserts that the proficiency of teachers is determined by knowledge, expertise and routine, which increases, with experience. We believe that in the present digital instruction framework the extent and quality of teachers' digital competence is of vital importance in addition to their ability to cope with the new challenges, meet the respective demands, support their students and transmit the given knowledge.

The dynamic development of telematics, computer technology and the information and communication technologies resulted in the emergence of the knowledge and information-centred society [8]. The main beneficiaries of these trends are those who are motivated toward knowledge acquisition and capable of self-directed learning. The learning process enriched with the three dimensional augmented reality giving rise to an alternative spatial awareness provides a special experience for students. Such approaches utilize the latest ICT-based and Cognitive Infocommunications methodologies including the virtual classroom and VR systems (google class, classcraft), interactive digital educational materials or human-computer interaction based methods [9], for example.

Several authors, among them Lajos Besenyei emphasized that the information society leads "to the emergence of a fully different social co-existence system." [10] The symbol of our age is the computer which became fully integrated into everyday life. The network contributed to the "constriction of space and the elimination of distance as the virtual world spans over the Earth and connections can be established in seconds between people living at the farthest points of our planet." Consequently, unprecedented learning formats and options appeared [10]. Specific devices notwithstanding and with general consequences in mind, we can 
declare that the prevalence of info-communication technology in education generated a substantial impact on the formation of human relationship networks. Accordingly, the learning, problem solving, decision making and mutual support options facilitated by increasingly diverse networks have undergone substantial change. The radical transformation of social networks, enabled citizens to expand their previously, close-knit, communities, achieve more variety in their connections and obtain more efficient forms of information acquisition. The online framework or world resulted in unprecedented communication potential and information gathering capacity enabling people or users to appear, act, communicate, or develop new methods of keeping touch independently or as part of a community [11]. Due to the prevalence of internet-based communication and medialization education faces several challenges in the present and in the future. The tripartite Prussian instruction paradigm emphasizing reading, writing, and arithmetic introduced in the eighteenth century is superseded by the $4 \mathrm{C}$ (Collaboration, Curiosity, Complexity and Creativity) model. As László Z. Karvalics argues, "influenced by the postmodern scepticism toward the fragmentation of knowledge brought on by the lack of transparency in the given disciplines and the mistrust toward practical activities requiring specialized skills a general lack of credibility can be discerned." [12]. Finding or providing information via links, the ability to search in digitally recorded texts, and the easy way to copy lead to radical changes in established academic practice facilitating editing open texts and plagiarism, at the same time.

The theoretical basis behind educational networks, includes the concept of connectivism, representing a cross-section between informatics, pedagogy, and network research promoted by George Siemens [13]. The theory holds that internet-based communication and media supported information exchange play a central role in the informal network context. Such information exchange is part of lifelong learning schemes and it differs from its previous counterparts as among others it can be embedded into free time activities and motivation continues to be one of its crucial requirements. As Sándor Forgó asserted "connectivism should be considered an integrated approach while focusing on the key components of instruction including competence, content, motivation, flexibility, and the evaluation of learner progress." [14]

The full integration of info-communication methods [15] [16] like mathability [17] [18] or other HMI based interfaces [19] [20] into everyday practice has led to increasing medialization of and the prevalence of digital technology in education [21] to detect learning problems [22] [23] or improve student's skills like problem solving [24]. The use of ICT in the education process boosted by the emergence of the worldwide web led to several tendencies and trends including the prevalence of digital data recording, the penetration of computers in the private sphere, followed by the world of work and learning, and eventually everyday life. Some of these factors have already reached the plateau of productivity. Based on the 
above, the results of the study related to digital education in the comparison of Serbia, Slovakia and Hungary are presented in this article.

Based on the above, the results of the study, related to the digital education, comparing Serbia, Slovakia and Hungary, are presented in this article.

\section{Methodology}

At the end of March 2020, two weeks after the nationwide conversion to digital instruction, we launched an online questionnaire-based survey, focusing on the initial experiences of pedagogues related to digital instruction and their respective role, competences and attitudes in three countries, Hungary, Slovakia and Serbia. The data for the inquiry was obtained via simple and random sampling. The samples were compiled by the snowball approach as the potential respondents were contacted and informed on social media and through personal connections concerning the objectives of the research program and the guarantee of anonymity. Therefore the sample cannot be considered representative. Following the three week period allotted for the completion of the questionnaires the results were systemised, purified, and coded. The survey resulted in 3390, 141, and 193 valid responses from Hungary, Serbia, and Slovakia respectively. The data were processed via difference statistical approach using SPSS 23 software. We resorted to the triangulation method, in order to explore and compare the ICT use habits and pedagogical attitudes. This method based on difference statistics facilitates a multifaceted inquiry of the current situation, the digital competences of educators, the willingness of pedagogues to apply ICT devices on their own, and the methods and experiences related to ICT use. Since our research efforts focused on a heretofore unexplored field, due to the lack of relevant research results and experiences, we were not able to arrive at any unequivocal conclusions. Consequently, we tested null-hypotheses. The individual variables were assessed by the Likert scale considering the former ordinal variables. We relied on two statistical analysis methods. During the processing of two partial samples (male/female, participated in training/didn't participate in training) we used the Mann-Whitney probe, while in cases when more than two sample components (highest educational level) had to be compared we resorted to the Kruskal-Wallis test. Due to spatial and length related requirements and for the sake of clarity the statistical test results (ranking scores, p, U, Chi square values, average figures) are not included in the text, but we list them in separate tables related to the given hypotheses. In order to observe the extent and content-based restrictions of the study we provide a detailed description of only the most informative results of our research effort. 


\section{Results and Discussion}

The next charts illustrate the country-specific distribution of pedagogues completing the questionnaire according to the respective background variables. The majority of respondents in all three countries were women in (Serbia 60\%, Slovakia $77.5 \%$ and Hungary $82.4 \%$ ) respectively.

We have assessed the educational background and qualification of the respondents. While there are significant differences concerning the size of the sample, it was revealed that the Hungarian participants had the lowest level of qualification as the number of those with MA/MSc degree and $\mathrm{PhD}$ lags behind that of their counterparts from the other two countries (Figure 1).

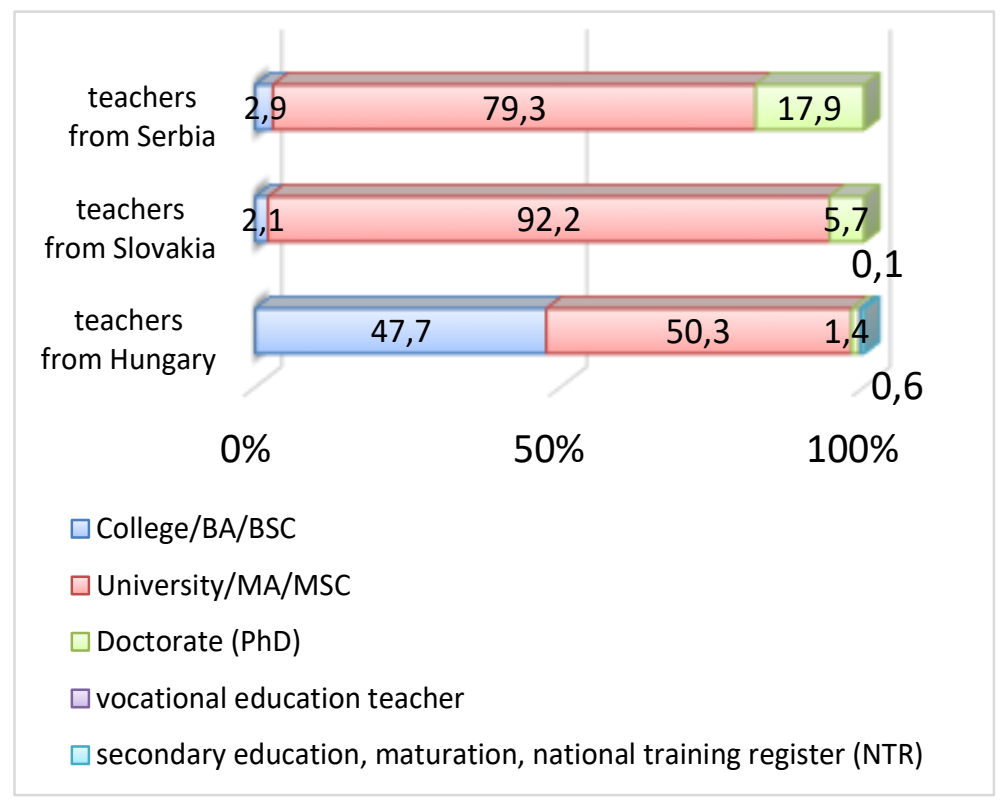

Figure 1

Highest education level of respondents

Prior to launching the research project we hypothesized that familiarity with digital pedagogy could generate a significant impact on ICT use. Consequently, we assessed what percentage of the respondents received digital training during their studies and how many respondents took part in such training outside the framework of their studies. While only a small portion $(20.7 \%)$, of Serbian colleagues, received digital training during their studies, it is encouraging that more than half of them felt it was needed and made up for this deficiency in an extracurricular fashion. In the same vein, although half (46.6\%) of Slovakian teachers participated in digital pedagogy training during their studies, the high rate of extracurricular participation in such programs demonstrates the priority 
assigned to digital instruction. Similar results can be seen in case of Hungary as more than half $(50.3 \%)$ of the respondents participated in digital training during their studies, but they took part in such programs in an extracurricular format as well.

Assessing the availability of digital devices for the pedagogues in the participating countries was a crucial component of the research program. The respective rates were favourable in all three countries (Figure 2). The highest access level was shown in Slovakia, while the lowest scores were in Serbia.

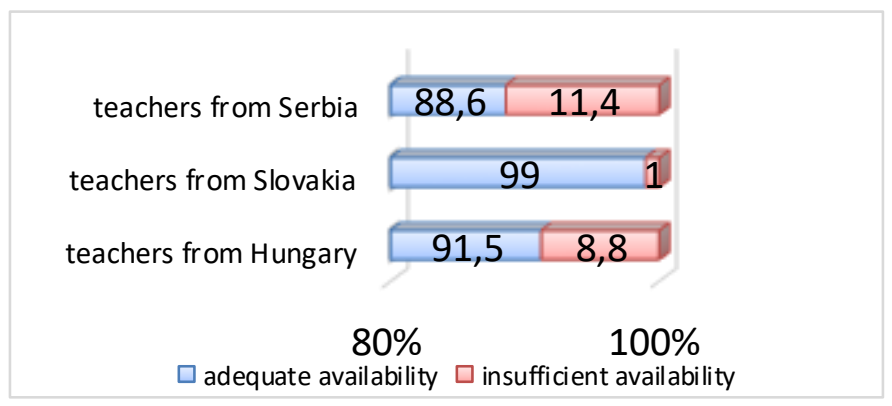

Figure 2

Digital equipment availability

The results of the specific statistical analyses are summarized below in the form of tables (Table 1-13).

Table 1

The distribution of ICT use and attitudes according to the participating countries

\begin{tabular}{|c|c|c|c|c|c|c|c|}
\hline & & $\begin{array}{l}\text { Hungary } \\
\%\end{array}$ & $\begin{array}{l}\text { Hungarian } \\
\text { average }\end{array}$ & $\begin{array}{l}\text { Slovakian } \\
\%\end{array}$ & $\begin{array}{l}\text { Slovakian } \\
\text { average }\end{array}$ & $\begin{array}{l}\text { Serbian } \\
\%\end{array}$ & $\begin{array}{l}\text { Serbian } \\
\text { average }\end{array}$ \\
\hline \multirow{6}{*}{$\begin{array}{l}\text { Possession of basic } \\
\text { ICT skills }\end{array}$} & 1 & 1.1 & \multirow[t]{6}{*}{4.53} & 4.1 & \multirow[t]{6}{*}{4.86} & 2.1 & \multirow[t]{6}{*}{5.22} \\
\hline & 2 & 5.2 & & 1.0 & & 2.9 & \\
\hline & 3 & 17.6 & & 10.9 & & 9.3 & \\
\hline & 4 & 20.1 & & 17.1 & & 5.7 & \\
\hline & 5 & 28.1 & & 22.3 & & 16.4 & \\
\hline & 6 & 28.0 & & 44.6 & & 63.6 & \\
\hline \multirow{6}{*}{$\begin{array}{l}\text { Confident } \\
\text { application of ICT } \\
\text { during teaching }\end{array}$} & 1 & 2.3 & \multirow[t]{6}{*}{4.27} & 3.6 & \multirow[t]{6}{*}{4.87} & 0 & \multirow[t]{6}{*}{5.07} \\
\hline & 2 & 6.8 & & 2.6 & & 5.7 & \\
\hline & 3 & 20.1 & & 5.2 & & 8.6 & \\
\hline & 4 & 23.6 & & 20.2 & & 11.4 & \\
\hline & 5 & 26.9 & & 29.0 & & 21.4 & \\
\hline & 6 & 20.3 & & 39.4 & & 52.9 & \\
\hline \multirow{6}{*}{ 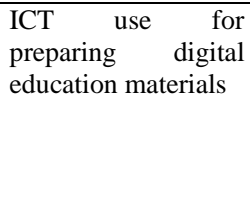 } & 1 & 5.5 & \multirow[t]{6}{*}{4.04} & 3.1 & \multirow[t]{6}{*}{4.68} & 2.1 & \multirow[t]{6}{*}{5.11} \\
\hline & 2 & 11.7 & & 4.7 & & 2.9 & \\
\hline & 3 & 19.2 & & 10.9 & & 8.6 & \\
\hline & 4 & 20.3 & & 18.7 & & 12.9 & \\
\hline & 5 & 22.4 & & 28.0 & & 15.0 & \\
\hline & 6 & 20.7 & & 34.7 & & 58.6 & \\
\hline
\end{tabular}




\begin{tabular}{|c|c|c|c|c|c|c|c|}
\hline \multirow{6}{*}{$\begin{array}{l}\text { Supporting } r \\
\text { learning the } \\
\text { through digital } \\
\text { communication } \\
\text { options }\end{array}$} & 1 & 1.8 & \multirow[t]{6}{*}{4.67} & 3.1 & \multirow[t]{6}{*}{5.04} & 0 & \multirow[t]{6}{*}{5.23} \\
\hline & 2 & 6.0 & & 1.6 & & 5.7 & \\
\hline & 3 & 12.1 & & 7.3 & & 5.0 & \\
\hline & 4 & 17.4 & & 13.0 & & 9.3 & \\
\hline & 5 & 28.6 & & 26.9 & & 20.7 & \\
\hline & 6 & 34.1 & & 48.2 & & 59.3 & \\
\hline \multirow{6}{*}{$\begin{array}{lr}\text { ICT use for } \\
\text { monitoring } \\
\text { evaluation }\end{array}$} & 1 & 8.2 & \multirow[t]{6}{*}{3.93} & 6.7 & \multirow[t]{6}{*}{4.43} & 1.4 & \multirow[t]{6}{*}{4.91} \\
\hline & 2 & 12.9 & & 5.2 & & 7.9 & \\
\hline & 3 & 17.3 & & 11.9 & & 8.6 & \\
\hline & 4 & 19.8 & & 20.7 & & 11.4 & \\
\hline & 5 & 23.3 & & 25.4 & & 22.1 & \\
\hline & 6 & 18.6 & & 30.1 & & 48.6 & \\
\hline \multirow{6}{*}{$\begin{array}{l}\text { Simultaneous use of } \\
\text { several ICT services }\end{array}$} & 1 & 8.4 & \multirow[t]{6}{*}{4.10} & 4.1 & \multirow[t]{6}{*}{4.54} & 5.0 & \multirow[t]{6}{*}{4.89} \\
\hline & 2 & 11.3 & & 6.7 & & 5.7 & \\
\hline & 3 & 14.5 & & 9.8 & & 9.3 & \\
\hline & 4 & 17.3 & & 23.8 & & 10.7 & \\
\hline & 5 & 24.3 & & 21.2 & & 13.6 & \\
\hline & 6 & 24.2 & & 34.2 & & 55.7 & \\
\hline \multirow{6}{*}{$\begin{array}{l}\text { Only uses reliable } \\
\text { systems }\end{array}$} & 1 & 1.6 & \multirow[t]{6}{*}{4.88} & 2.1 & \multirow[t]{6}{*}{4.979} & 2.9 & \multirow[t]{6}{*}{4.97} \\
\hline & 2 & 4.3 & & 3.1 & & 4.3 & \\
\hline & 3 & 9.9 & & 6.2 & & 7.1 & \\
\hline & 4 & 13.7 & & 16.6 & & 12.9 & \\
\hline & 5 & 29.9 & & 27.5 & & 24.3 & \\
\hline & 6 & 40.7 & & 44.6 & & 48.6 & \\
\hline
\end{tabular}

The ICT use habits were assessed on a six step Likert scale with 1 indicating total rejection and 6 standing for absolute acceptance. The table reveals that the highest scores and average values were discerned in case of Serbian teachers. This result is especially noteworthy as the rate of participation in digital training either during one's studies or in an extracurricular manner was the lowest with Serbian respondents, not to mention that the availability of digital devices was the lowest in their case. The lowest overall scores, however, were by Hungarian participants.

Table 2

Correlation of ICT use among Hungarian pedagogues

\begin{tabular}{|c|c|c|c|c|c|c|}
\hline $\begin{array}{l}\text { Dependent } \\
\text { variable }\end{array}$ & $\begin{array}{l}\text { Independent } \\
\text { variable: sex }\end{array}$ & MR & $\mathrm{U}$ & $\mathrm{p}$ & average & group median \\
\hline \multirow{2}{*}{$\begin{array}{l}\text { Possession of } \\
\text { basic ICT skills }\end{array}$} & Male & 1997.64 & \multirow[t]{2}{*}{836832} & \multirow[t]{2}{*}{$<0.000$} & 4.85 & 5.11 \\
\hline & Female & 1652.75 & & & 4.44 & 4.54 \\
\hline \multirow{2}{*}{$\begin{array}{l}\text { Confident } \\
\text { application of } \\
\text { ICT during } \\
\text { teaching }\end{array}$} & Male & 2062.58 & \multirow[t]{2}{*}{786176.5} & \multirow[t]{2}{*}{$<0.000$} & 4.69 & 4.91 \\
\hline & Female & 1633.85 & & & 4.15 & 4.21 \\
\hline \multirow{2}{*}{$\begin{array}{l}\text { ICT use for } \\
\text { preparing digital } \\
\text { education } \\
\text { materials }\end{array}$} & Male & 1993.92 & \multirow[t]{2}{*}{839734.5} & \multirow[t]{2}{*}{$<0.000$} & 4.43 & 4.67 \\
\hline & Female & 1653.83 & & & 3.93 & 4.01 \\
\hline \multirow[b]{2}{*}{$\begin{array}{l}\text { Supporting the } \\
\text { learning process } \\
\text { through digital } \\
\text { communication } \\
\text { options }\end{array}$} & Male & 1812.38 & \multirow[t]{2}{*}{981336} & \multirow[t]{2}{*}{$<0.000$} & 4.76 & 5.07 \\
\hline & Female & 1706.67 & & & 4.65 & 4.88 \\
\hline
\end{tabular}




\begin{tabular}{|c|c|c|c|c|c|c|}
\hline \multirow{2}{*}{$\begin{array}{lr}\text { ICT use for } \\
\text { monitoring and } \\
\text { evaluation }\end{array}$} & Male & 1946.02 & \multirow[t]{2}{*}{877092} & \multirow[t]{2}{*}{$<0.000$} & 4.26 & 4.49 \\
\hline & Female & 1667.77 & & & 3.83 & 3.95 \\
\hline \multirow{2}{*}{$\begin{array}{l}\text { Simultaneous } \\
\text { use of several } \\
\text { ICT services }\end{array}$} & Male & 1898.41 & \multirow[t]{2}{*}{914228.5} & \multirow[t]{2}{*}{$<0.000$} & 4.35 & 4.71 \\
\hline & Female & 1681.63 & & & 4.03 & 4.24 \\
\hline \multirow{2}{*}{$\begin{array}{l}\text { Only uses } \\
\text { reliable systems }\end{array}$} & Male & 1738.78 & \multirow[t]{2}{*}{1038743.5} & \multirow[t]{2}{*}{0.782} & 4.91 & 5.16 \\
\hline & Female & 1728.09 & & & 4.87 & 5.16 \\
\hline
\end{tabular}

Table 3

Correlation of ICT use among Slovakian pedagogues

\begin{tabular}{|c|c|c|c|c|c|c|}
\hline $\begin{array}{l}\text { Dependent } \\
\text { variable }\end{array}$ & $\begin{array}{l}\text { Independent } \\
\text { variable: sex }\end{array}$ & MR & $\mathrm{U}$ & $\mathrm{p}$ & average & $\begin{array}{l}\text { group } \\
\text { median }\end{array}$ \\
\hline \multirow{2}{*}{$\begin{array}{l}\text { Possession of } \\
\text { basic ICT skills }\end{array}$} & Male & 106.25 & \multirow[t]{2}{*}{2388.5} & \multirow[t]{2}{*}{0.261} & 5.00 & 5.40 \\
\hline & Female & 95.02 & & & 4.83 & 5.12 \\
\hline \multirow{2}{*}{$\begin{array}{l}\text { Confident } \\
\text { application of ICT } \\
\text { during teaching }\end{array}$} & Male & 91.43 & \multirow[t]{2}{*}{2513.5} & \multirow[t]{2}{*}{0.501} & 4.65 & 4.94 \\
\hline & Female & 98.19 & & & 4.91 & 5.14 \\
\hline \multirow{2}{*}{$\begin{array}{lr}\text { ICT use for } \\
\text { preparing digital } \\
\text { education } \\
\text { materials }\end{array}$} & Male & 93.03 & \multirow[t]{2}{*}{2568} & \multirow[t]{2}{*}{0.636} & 4.50 & 4.83 \\
\hline & Female & 97.85 & & & 4.72 & 4.96 \\
\hline \multirow[b]{2}{*}{$\begin{array}{l}\text { Supporting the } \\
\text { learning process } \\
\text { through digital } \\
\text { communication } \\
\text { options }\end{array}$} & Male & 85.66 & \multirow[t]{2}{*}{2317.5} & \multirow[t]{2}{*}{0.161} & 4.62 & 5.13 \\
\hline & Female & 99.42 & & & 5.13 & 5.34 \\
\hline \multirow{2}{*}{$\begin{array}{lr}\text { ICT use for } \\
\text { monitoring } \\
\text { evaluation }\end{array}$} & Male & 92.71 & \multirow[t]{2}{*}{2557} & \multirow[t]{2}{*}{0.612} & 4.24 & 4.60 \\
\hline & Female & 97.92 & & & 4.47 & 4.70 \\
\hline \multirow{2}{*}{$\begin{array}{l}\text { Simultaneous use } \\
\text { of several ICT } \\
\text { services }\end{array}$} & Male & 92.91 & \multirow[t]{2}{*}{2564} & \multirow[t]{2}{*}{0.627} & 4.44 & 4.65 \\
\hline & Female & 97.87 & & & 4.56 & 4.80 \\
\hline \multirow{2}{*}{$\begin{array}{l}\text { Only uses reliable } \\
\text { systems }\end{array}$} & Male & 74.46 & \multirow[t]{2}{*}{1936.5} & \multirow[t]{2}{*}{0.006} & 4.382 & 4.688 \\
\hline & Female & 101.82 & & & 5.107 & 5.311 \\
\hline
\end{tabular}

Table 4

Correlation of ICT use among Serbian pedagogues

\begin{tabular}{|c|c|c|c|c|c|c|}
\hline \multicolumn{7}{|c|}{ Serbian - Mann-Whitney-probe: Correlation between sex and teachers' ICT use } \\
\hline $\begin{array}{l}\text { Dependent } \\
\text { variable }\end{array}$ & $\begin{array}{l}\text { Independent } \\
\text { variable: sex }\end{array}$ & MR & $\mathrm{U}$ & $\mathrm{p}$ & average & $\begin{array}{l}\text { group } \\
\text { median }\end{array}$ \\
\hline \multirow{2}{*}{$\begin{array}{l}\text { Possession of } \\
\text { basic ICT skills }\end{array}$} & Male & 71.25 & \multirow[t]{2}{*}{2310.000000} & \multirow[t]{2}{*}{0.835} & 5.25 & 5.57 \\
\hline & Female & 70.00 & & & 5.20 & 5.53 \\
\hline \multirow{2}{*}{$\begin{array}{l}\text { Confident } \\
\text { application of } \\
\text { ICT during } \\
\text { teaching }\end{array}$} & Male & 74.93 & \multirow[t]{2}{*}{2104.000000} & \multirow[t]{2}{*}{0.250} & 5.20 & 5.48 \\
\hline & Female & 67.55 & & & 4.99 & 5.28 \\
\hline \multirow{2}{*}{$\begin{array}{l}\text { ICT use for } \\
\text { preparing digital } \\
\text { education } \\
\text { materials }\end{array}$} & Male & 73.50 & \multirow[t]{2}{*}{2184.000000} & \multirow[t]{2}{*}{0.422} & 5.20 & 5.51 \\
\hline & Female & 68.50 & & & 5.06 & 5.38 \\
\hline
\end{tabular}




\begin{tabular}{|c|c|c|c|c|c|c|}
\hline \multirow{2}{*}{$\begin{array}{l}\text { Supporting the } \\
\text { learning process } \\
\text { through digital } \\
\text { communication } \\
\text { options }\end{array}$} & Male & 70.27 & \multirow[t]{2}{*}{2339.000000} & \multirow[t]{2}{*}{0.950} & 5.21 & 5.49 \\
\hline & Female & 70.65 & & & 5.24 & 5.49 \\
\hline \multirow{2}{*}{$\begin{array}{lr}\text { ICT use for } \\
\text { monitoring and } \\
\text { evaluation }\end{array}$} & Male & 70.02 & \multirow[t]{2}{*}{2325.000000} & \multirow[t]{2}{*}{0.902} & 4.86 & 5.29 \\
\hline & Female & 70.82 & & & 4.94 & 5.26 \\
\hline \multirow{2}{*}{$\begin{array}{l}\text { Simultaneous use } \\
\text { of several ICT } \\
\text { services }\end{array}$} & Male & 73.54 & \multirow[t]{2}{*}{2181.500000} & \multirow[t]{2}{*}{0.424} & 4.96 & 5.45 \\
\hline & Female & 68.47 & & & 4.85 & 5.30 \\
\hline \multirow{2}{*}{$\begin{array}{l}\text { Only uses } \\
\text { reliable systems }\end{array}$} & Male & 70.07 & \multirow[t]{2}{*}{2328.000000} & \multirow[t]{2}{*}{0.913} & 4.93 & 5.30 \\
\hline & Female & 70.79 & & & 5.00 & 5.29 \\
\hline
\end{tabular}

We relied on the Mann-Whitney proble to identify significant divergences between answers provided by women and men regarding ICT use. (scores indicating significance are highlighted with blue) It is noteworthy that in case of Hungarian teachers we could discern significant divergence between the sexes with the exception of the question concerning the use of the reliable systems. Regardless of significance men received higher scores. Only one significant digression could be revealed among pedagogues from Slovakia as women achieved higher scores despite the self-professed higher ICT skills by men. The significant digression, favouring women, was identified in case of the use of reliable systems. Responses by Serbian teachers are more diverse as the scores differ according to sex in some questions, while no significant digression could be identified.

Table 5

ICT use in light of digital training among Hungarian teachers

\begin{tabular}{|c|c|c|c|c|c|c|}
\hline \multicolumn{7}{|c|}{$\begin{array}{l}\text { Hungarian- Mann-Whitney-probe: Correlation between digital training received during studies and } \\
\text { teachers' ICT use }\end{array}$} \\
\hline $\begin{array}{l}\text { Dependent } \\
\text { variable }\end{array}$ & $\begin{array}{l}\text { Independent } \\
\text { variable: } \\
\text { digital } \\
\text { training } \\
\text { during } \\
\text { studies }\end{array}$ & $\mathrm{MR}$ & $\mathrm{U}$ & $\mathrm{p}$ & average & $\begin{array}{l}\text { group } \\
\text { median }\end{array}$ \\
\hline \multirow{2}{*}{$\begin{array}{l}\text { Possession of } \\
\text { basic ICT skills }\end{array}$} & no & 1559.50 & \multirow[t]{2}{*}{1202440} & \multirow[t]{2}{*}{$<0.000$} & 4.30 & 4.38 \\
\hline & yes & 1899.34 & & & 4.76 & 4.94 \\
\hline \multirow{2}{*}{$\begin{array}{l}\text { Confident } \\
\text { application of } \\
\text { ICT during } \\
\text { teaching }\end{array}$} & no & 1535.38 & \multirow[t]{2}{*}{1160974.5} & \multirow[t]{2}{*}{$<0.000$} & 4.00 & 4.03 \\
\hline & yes & 1923.16 & & & 4.53 & 4.64 \\
\hline \multirow{2}{*}{$\begin{array}{l}\text { ICT use for } \\
\text { preparing digital } \\
\text { education } \\
\text { materials }\end{array}$} & no & 1527.45 & \multirow[t]{2}{*}{1147355} & \multirow[t]{2}{*}{$<0.000$} & 3.73 & 3.75 \\
\hline & yes & 1930.98 & & & 4.36 & 4.51 \\
\hline \multirow[b]{2}{*}{$\begin{array}{l}\text { Supporting the } \\
\text { learning process } \\
\text { through digital } \\
\text { communication } \\
\text { options }\end{array}$} & no & 1604.51 & \multirow[t]{2}{*}{1279817.5} & \multirow[t]{2}{*}{$<0.000$} & 4.49 & 4.71 \\
\hline & yes & 1854.90 & & & 4.85 & 5.10 \\
\hline
\end{tabular}




\begin{tabular}{|c|c|c|c|c|c|c|}
\hline \multirow{2}{*}{$\begin{array}{l}\text { ICT use for } \\
\text { monitoring and } \\
\text { evaluation }\end{array}$} & no & 1563.49 & \multirow[t]{2}{*}{1209302.5} & \multirow[t]{2}{*}{$<0.000$} & 3.66 & 3.72 \\
\hline & yes & 1895.40 & & & 4.20 & 4.38 \\
\hline \multirow{2}{*}{$\begin{array}{l}\text { Simultaneous } \\
\text { use of several } \\
\text { ICT services }\end{array}$} & no & 1569.93 & \multirow[t]{2}{*}{1220363} & \multirow[t]{2}{*}{$<0.000$} & 3.82 & 4.04 \\
\hline & yes & 1889.04 & & & 4.38 & 4.61 \\
\hline \multirow{2}{*}{$\begin{array}{l}\text { Only } \quad \text { uses } \\
\text { reliable }\end{array}$} & no & 1675.59 & \multirow[t]{2}{*}{1401991} & \multirow[t]{2}{*}{0.001} & 4.79 & 5.10 \\
\hline & yes & 1784.72 & & & 4.96 & 5.21 \\
\hline
\end{tabular}

Table 6

ICT use in light of digital training among Slovakian teachers

\begin{tabular}{|c|c|c|c|c|c|c|}
\hline \multicolumn{7}{|c|}{$\begin{array}{l}\text { Slovakian- Mann-Whitney probe: Correlation between digital training received during studies and } \\
\text { teachers' ICT use }\end{array}$} \\
\hline $\begin{array}{l}\text { Dependent } \\
\text { variable }\end{array}$ & $\begin{array}{l}\text { Independent } \\
\text { variable: } \\
\text { digital } \\
\text { training } \\
\text { during } \\
\text { studies }\end{array}$ & MR & $\mathrm{U}$ & $\mathrm{p}$ & average & $\begin{array}{l}\text { group } \\
\text { median }\end{array}$ \\
\hline \multirow{2}{*}{$\begin{array}{l}\text { Possession of } \\
\text { basic ICT skills }\end{array}$} & no & 86.27 & \multirow[t]{2}{*}{3529.500000} & \multirow[t]{2}{*}{0.003} & 4.63 & 4.86 \\
\hline & yes & 109.28 & & & 5.12 & 5.41 \\
\hline \multirow{2}{*}{$\begin{array}{l}\text { Confident } \\
\text { application of } \\
\text { ICT during } \\
\text { teaching }\end{array}$} & no & 90.82 & \multirow[t]{2}{*}{3998.500000} & \multirow[t]{2}{*}{0.084} & 4.74 & 4.98 \\
\hline & yes & 104.07 & & & 5.01 & 5.25 \\
\hline \multirow{2}{*}{$\begin{array}{l}\text { ICT use for } \\
\text { preparing digital } \\
\text { education } \\
\text { materials } \\
\end{array}$} & no & 89.93 & \multirow[t]{2}{*}{3907.000000} & \multirow[t]{2}{*}{0.051} & 4.52 & 4.72 \\
\hline & yes & 105.09 & & & 4.86 & 5.15 \\
\hline \multirow[b]{2}{*}{$\begin{array}{l}\text { Supporting the } \\
\text { learning process } \\
\text { through digital } \\
\text { communication } \\
\text { options }\end{array}$} & no & 99.03 & \multirow[t]{2}{*}{4426.000000} & \multirow[t]{2}{*}{0.562} & 5.09 & 5.34 \\
\hline & yes & 94.68 & & & 4.98 & 5.27 \\
\hline \multirow{2}{*}{$\begin{array}{lr}\text { ICT use for } \\
\text { monitoring and } \\
\text { evaluation }\end{array}$} & no & 90.76 & \multirow[t]{2}{*}{3992.500000} & \multirow[t]{2}{*}{0.088} & 4.25 & 4.53 \\
\hline & yes & 104.14 & & & 4.63 & 4.88 \\
\hline \multirow{2}{*}{$\begin{array}{l}\text { Simultaneous use } \\
\text { of several ICT } \\
\text { services }\end{array}$} & no & 89.21 & \multirow[t]{2}{*}{3833.000000} & \multirow[t]{2}{*}{0.032} & 4.32 & 4.53 \\
\hline & yes & 105.91 & & & 4.79 & 5.02 \\
\hline \multirow{2}{*}{$\begin{array}{l}\text { Only uses } \\
\text { reliable systems }\end{array}$} & no & 97.67 & \multirow[t]{2}{*}{4566.500000} & \multirow[t]{2}{*}{0.851} & 5.00 & 5.247 \\
\hline & yes & 96.24 & & & 4.956 & 5.21 \\
\hline
\end{tabular}

Table 7

ICT use in light of digital training among Serbian teachers

\begin{tabular}{|l|l|l|l|l|l|l|}
\hline $\begin{array}{l}\text { Serbian- Mann-Whitney probe: Correlation between digital training received during studies and } \\
\text { teachers' ICT use }\end{array}$ \\
\hline $\begin{array}{l}\text { Dependent } \\
\text { variable }\end{array}$ & $\begin{array}{l}\text { Independent } \\
\text { variable } \\
\text { digital } \\
\text { training } \\
\text { during } \\
\text { studies }\end{array}$ & MR & U & $\mathrm{p}$ & average & $\begin{array}{l}\text { group } \\
\text { median }\end{array}$ \\
& no & 68.13 & 1346.500000 & 0.115 & 5.14 & 5.49 \\
\cline { 2 - 6 } & yes & 79.57 & & & 5.52 & 5.72 \\
\hline
\end{tabular}




\begin{tabular}{|c|c|c|c|c|c|c|}
\hline \multirow{2}{*}{$\begin{array}{l}\text { Confident } \\
\text { application of } \\
\text { ICT during } \\
\text { teaching }\end{array}$} & no & 67.26 & \multirow[t]{2}{*}{1249.500000} & \multirow[t]{2}{*}{0.043} & 4.96 & 5.29 \\
\hline & yes & 82.91 & & & 5.48 & 5.63 \\
\hline \multirow{2}{*}{$\begin{array}{ll}\text { ICT use for } \\
\text { preparing } \\
\text { digital } \\
\text { education } \\
\text { materials } \\
\end{array}$} & no & 67.32 & \multirow[t]{2}{*}{1256.500000} & \multirow[t]{2}{*}{0.042} & 5.01 & 5.35 \\
\hline & yes & 82.67 & & & 5.52 & 5.71 \\
\hline \multirow[b]{2}{*}{$\begin{array}{l}\text { Supporting the } \\
\text { learning } \\
\text { process through } \\
\text { digital } \\
\text { communication } \\
\text { options }\end{array}$} & no & 67.66 & \multirow[t]{2}{*}{1294.000000} & \multirow[t]{2}{*}{0.067} & 5.13 & 5.43 \\
\hline & yes & 81.38 & & & 5.62 & 5.69 \\
\hline \multirow{2}{*}{$\begin{array}{l}\text { ICT use for } \\
\text { monitoring and } \\
\text { evaluation }\end{array}$} & no & 68.18 & \multirow[t]{2}{*}{1352.000000} & \multirow[t]{2}{*}{0.156} & 4.81 & 5.22 \\
\hline & yes & 79.38 & & & 5.28 & 5.45 \\
\hline \multirow{2}{*}{$\begin{array}{l}\text { Simultaneous } \\
\text { use of several } \\
\text { ICT services }\end{array}$} & no & 67.60 & \multirow[t]{2}{*}{1287.500000} & \multirow[t]{2}{*}{0.068} & 4.77 & 5.28 \\
\hline & yes & 81.60 & & & 5.38 & 5.61 \\
\hline \multirow{2}{*}{$\begin{array}{l}\text { Only uses } \\
\text { reliable systems }\end{array}$} & no & 68.51 & \multirow[t]{2}{*}{1388.500000} & \multirow[t]{2}{*}{0.223} & 4.91 & 5.24 \\
\hline & yes & 78.12 & & & 5.21 & 5.48 \\
\hline
\end{tabular}

We relied on the Mann-Whitney probe to explore ICT use habits or in an indirect fashion we inquired about the efficiency of digital instruction, namely, whether receiving training in digital pedagogy during one's studies can have an impact on the effectiveness of teaching. Responses provided by Hungarian pedagogues receiving such training reveal a higher score and a significant divergence in case of all questions. Regarding the Slovakian responses, higher scores were given in case of two questions (Supporting the learning process through digital communication options, Only uses reliable systems) by those teachers, who did not take part in digital training. Consequently, two significant digressions can be identified regarding ICT skills and the ability to use several services simultaneously. Similarly to their Hungarian counterparts those Serbian teachers posted higher scores, who received digital training during their studies. Significant digressions were discerned regarding confident ICT use and the preparation of digital educational materials.

Table 8

ICT use in light of extracurricular digital training among Hungarian pedagogues

\begin{tabular}{|c|c|c|c|c|c|c|}
\hline \multicolumn{7}{|c|}{$\begin{array}{l}\text { Hungarian- Mann-Whitney-probe: Correlation between participation in digital pedagogy training and } \\
\text { teachers' ICT use }\end{array}$} \\
\hline $\begin{array}{l}\text { Dependent } \\
\text { variable }\end{array}$ & $\begin{array}{l}\text { Independent } \\
\text { variable: } \\
\text { extracurricular } \\
\text { digital } \\
\text { training }\end{array}$ & MR & $\mathrm{U}$ & $\mathrm{p}$ & average & $\begin{array}{l}\text { group } \\
\text { median }\end{array}$ \\
\hline \multirow{2}{*}{$\begin{array}{l}\text { Possession of } \\
\text { basic ICT skills }\end{array}$} & no & 1567.59 & \multirow[t]{2}{*}{1201416.5} & \multirow[t]{2}{*}{$<0.000$} & 4.29 & 4.41 \\
\hline & yes & 1833.09 & & & 4.68 & 4.82 \\
\hline \multirow{2}{*}{$\begin{array}{l}\text { Confident } \\
\text { application of }\end{array}$} & no & 1570.22 & \multirow[t]{2}{*}{1204926} & \multirow[t]{2}{*}{$<0.000$} & 4.04 & 4.10 \\
\hline & yes & 1831.44 & & & 4.41 & 4.50 \\
\hline
\end{tabular}




\begin{tabular}{|c|c|c|c|c|c|c|}
\hline $\begin{array}{l}\text { ICT during } \\
\text { teaching }\end{array}$ & & & & & & \\
\hline \multirow{2}{*}{$\begin{array}{ll}\text { ICT use for } \\
\text { preparing } & \\
\text { digital } & \\
\text { education } & \\
\text { materials } & \end{array}$} & no & 1560.69 & \multirow[t]{2}{*}{1192192} & \multirow[t]{2}{*}{$<0.000$} & 3.77 & 3.81 \\
\hline & yes & 1837.44 & & & 4.22 & 4.34 \\
\hline \multirow[b]{2}{*}{$\begin{array}{l}\text { Supporting the } \\
\text { learning } \\
\text { process through } \\
\text { digital } \\
\text { communication } \\
\text { options }\end{array}$} & no & 1657.611776 & \multirow[t]{2}{*}{1321772.5} & \multirow[t]{2}{*}{$<0.000$} & 4.55 & 4.81 \\
\hline & yes & & & & 4.75 & 5.00 \\
\hline \multirow{2}{*}{$\begin{array}{l}\text { ICT use for } \\
\text { monitoring and } \\
\text { evaluation }\end{array}$} & no & 1614.75 & \multirow[t]{2}{*}{1264465.5} & \multirow[t]{2}{*}{$<0.000$} & 3.73 & 3.84 \\
\hline & yes & 1803.40 & & & 4.05 & 4.21 \\
\hline \multirow{2}{*}{$\begin{array}{l}\text { Simultaneous } \\
\text { use of several } \\
\text { ICT services }\end{array}$} & no & 1594.64 & \multirow[t]{2}{*}{1237578} & \multirow[t]{2}{*}{$<0.000$} & 3.86 & 4.05 \\
\hline & yes & 1816.06 & & & 4.25 & 4.49 \\
\hline \multirow{2}{*}{$\begin{array}{l}\text { Only uses } \\
\text { reliable systems }\end{array}$} & no & 1683.06 & \multirow[t]{2}{*}{1355803} & \multirow[t]{2}{*}{0.020} & 4.77 & 5.12 \\
\hline & yes & 1760.37 & & & 4.95 & 5.18 \\
\hline
\end{tabular}

Table 9

ICT use in light of digital training among Slovakian pedagogues

\begin{tabular}{|c|c|c|c|c|c|c|}
\hline \multicolumn{7}{|c|}{$\begin{array}{l}\text { Slovakian- Mann-Whitney-probe: Correlation between participation in extracurricular digita } \\
\text { pedagogy training and teachers' ICT use }\end{array}$} \\
\hline $\begin{array}{l}\text { Dependent } \\
\text { variable }\end{array}$ & $\begin{array}{l}\text { Independent } \\
\text { variable: } \\
\text { extracurricular } \\
\text { digital training }\end{array}$ & MR & $\mathrm{U}$ & $\mathrm{p}$ & average & $\begin{array}{l}\text { group } \\
\text { median }\end{array}$ \\
\hline \multirow{2}{*}{$\begin{array}{l}\text { Possession of } \\
\text { basic ICT skills }\end{array}$} & no & 91.62 & \multirow[t]{2}{*}{2793.000000} & \multirow[t]{2}{*}{0.476} & 4.79 & 5.04 \\
\hline & yes & 98.36 & & & 4.88 & 4.91 \\
\hline \multirow{2}{*}{$\begin{array}{l}\text { Confident } \\
\text { application of } \\
\text { ICT during } \\
\text { teaching }\end{array}$} & no & 87.23 & \multirow[t]{2}{*}{2622.000000} & \multirow[t]{2}{*}{0.199} & 4.69 & 4.87 \\
\hline & yes & 99.47 & & & 4.91 & 5.17 \\
\hline \multirow{2}{*}{$\begin{array}{l}\text { ICT use for } \\
\text { preparing digital } \\
\text { education } \\
\text { materials }\end{array}$} & no & 89.00 & \multirow[t]{2}{*}{2691.000000} & \multirow[t]{2}{*}{0.299} & 4.51 & 4.63 \\
\hline & yes & 99.03 & & & 4.72 & 5.02 \\
\hline \multirow{2}{*}{$\begin{array}{l}\text { Supporting the } \\
\text { learning process } \\
\text { through digital } \\
\text { communication } \\
\text { options }\end{array}$} & no & 96.28 & \multirow[t]{2}{*}{2975.000000} & \multirow[t]{2}{*}{0.923} & 4.97 & 5.31 \\
\hline & yes & 97.18 & & & 5.05 & 5.31 \\
\hline \multirow{2}{*}{$\begin{array}{lr}\text { ICT use for } \\
\text { monitoring and } \\
\text { evaluation }\end{array}$} & no & 99.81 & \multirow[t]{2}{*}{2893.500000} & \multirow[t]{2}{*}{0.718} & 4.51 & 4.80 \\
\hline & yes & 96.29 & & & 4.41 & 4.65 \\
\hline \multirow{2}{*}{$\begin{array}{l}\text { Simultaneous use } \\
\text { of several ICT } \\
\text { services }\end{array}$} & no & 91.65 & \multirow[t]{2}{*}{2794.500000} & \multirow[t]{2}{*}{0.489} & 4.38 & 4.63 \\
\hline & yes & 98.35 & & & 4.58 & 4.81 \\
\hline \multirow{2}{*}{$\begin{array}{l}\text { Only uses } \\
\text { reliable systems }\end{array}$} & no & 100.96 & \multirow[t]{2}{*}{2848.500000} & \multirow[t]{2}{*}{0.598} & 5.154 & 5.281 \\
\hline & yes & 96.00 & & & 4.935 & 5.215 \\
\hline
\end{tabular}


Table 10

ICT use in light of extracurricular digital training among Serbian pedagogues

\begin{tabular}{|c|c|c|c|c|c|c|}
\hline $\begin{array}{l}\text { Dependent } \\
\text { variable }\end{array}$ & $\begin{array}{l}\text { Independent } \\
\text { variable } \\
\text { extracurricular } \\
\text { digital training }\end{array}$ & MR & $\mathrm{U}$ & $\mathrm{p}$ & average & $\begin{array}{l}\text { group } \\
\text { median }\end{array}$ \\
\hline \multirow{2}{*}{$\begin{array}{l}\text { Possession of } \\
\text { basic ICT skills }\end{array}$} & no & 67.09 & \multirow[t]{2}{*}{2210.500000} & \multirow[t]{2}{*}{0.294} & 5.17 & 5.47 \\
\hline & yes & 73.29 & & & 5.26 & 5.61 \\
\hline \multirow{2}{*}{$\begin{array}{l}\text { Confident } \\
\text { application of } \\
\text { ICT during } \\
\text { teaching }\end{array}$} & no & 67.96 & \multirow[t]{2}{*}{2265.500000} & \multirow[t]{2}{*}{0.465} & 5.05 & 5.28 \\
\hline & yes & 72.58 & & & 5.09 & 5.43 \\
\hline \multirow{2}{*}{$\begin{array}{ll}\text { ICT use for } \\
\text { preparing } \\
\text { digital } \\
\text { education } \\
\text { materials } \\
\end{array}$} & no & 68.04 & \multirow[t]{2}{*}{2270.500000} & \multirow[t]{2}{*}{0.466} & 5.02 & 5.38 \\
\hline & yes & 72.51 & & & 5.19 & 5.48 \\
\hline \multirow{2}{*}{$\begin{array}{l}\text { Supporting the } \\
\text { learning process } \\
\text { through digital } \\
\text { communication } \\
\text { options }\end{array}$} & no & 71.13 & \multirow[t]{2}{*}{2386.000000} & \multirow[t]{2}{*}{0.852} & 5.21 & 5.52 \\
\hline & yes & 69.99 & & & 5.25 & 5.47 \\
\hline \multirow{2}{*}{$\begin{array}{lr}\text { ICT use for } \\
\text { monitoring and } \\
\text { evaluation }\end{array}$} & no & 67.68 & \multirow[t]{2}{*}{2248.000000} & \multirow[t]{2}{*}{0.426} & 4.79 & 5.19 \\
\hline & yes & 72.81 & & & 5.00 & 5.33 \\
\hline \multirow{2}{*}{$\begin{array}{l}\text { Simultaneous } \\
\text { use of several } \\
\text { ICT services }\end{array}$} & no & 68.48 & \multirow[t]{2}{*}{2298.500000} & \multirow[t]{2}{*}{0.557} & 4.79 & 5.29 \\
\hline & yes & 72.15 & & & 4.97 & 5.41 \\
\hline \multirow{2}{*}{$\begin{array}{l}\text { Only uses } \\
\text { reliable systems }\end{array}$} & no & 68.10 & \multirow[t]{2}{*}{2274.500000} & \multirow[t]{2}{*}{0.497} & 4.83 & 5.23 \\
\hline & yes & 72.46 & & & 5.09 & 5.34 \\
\hline
\end{tabular}

Similarly to the previous test, this inquiry aimed to assess the impact of extracurricular digital training on the dependent variables. The responses by Hungarian teachers were similar to that of the training undertaken during one's studies as the participants gave significantly higher scores to all questions. Respondents from Slovakia, who did not participate in digital training beyond their studies provided higher ranking scores in connection with two questions (ICT use for monitoring and evaluation, Only uses reliable systems). While high scores were assigned to the other questions, none of the respective divergences can be considered significant. Serbian pedagogues participating in digital training regularly provided higher evaluations to the questions, but significant divergence could not be discerned in this category either. 
Table 11

ICT use in light of the highest level of education by Hungarian pedagogues

\begin{tabular}{|c|c|c|c|c|c|c|}
\hline $\begin{array}{l}\text { Dependent } \\
\text { variable }\end{array}$ & $\begin{array}{l}\text { Independent variable: } \\
\text { highest qualification }\end{array}$ & MR & $\begin{array}{l}\text { Chi- } \\
\text { square }\end{array}$ & $\mathrm{p}$ & average & $\begin{array}{l}\text { group } \\
\text { median }\end{array}$ \\
\hline \multirow{5}{*}{$\begin{array}{l}\text { Possession of } \\
\text { basic ICT skills }\end{array}$} & College/BA/BSC & 1613.28 & \multirow[t]{5}{*}{52.078} & \multirow[t]{5}{*}{$<0.000$} & 4.38 & 4.47 \\
\hline & University/MA/MSC & 1836.67 & & & 4.67 & 4.85 \\
\hline & $\mathrm{PhD}$ & 2004.07 & & & 4.81 & 5.18 \\
\hline & $\begin{array}{l}\text { Vocational education } \\
\text { teacher }\end{array}$ & 521.50 & & & 3.00 & 3.00 \\
\hline & $\begin{array}{l}\text { secondary } \\
\text { education/maturation/NTR }\end{array}$ & 1639.78 & & & 4.35 & 4.50 \\
\hline \multirow{5}{*}{$\begin{array}{l}\text { Confident } \\
\text { application of } \\
\text { ICT during } \\
\text { teaching }\end{array}$} & College/BA/BSC & 1629.90 & \multirow[t]{5}{*}{38.595} & \multirow[t]{5}{*}{$<0.000$} & 4.14 & 4.20 \\
\hline & University/MA/MSC & 1817.17 & & & 4.38 & 4.49 \\
\hline & $\mathrm{PhD}$ & 2006.04 & & & 4.60 & 4.82 \\
\hline & $\begin{array}{l}\text { Vocational education } \\
\text { teacher }\end{array}$ & 663.50 & & & 3.00 & 3.00 \\
\hline & $\begin{array}{l}\text { secondary } \\
\text { education/maturation/NTR }\end{array}$ & 1944.65 & & & 4.50 & 4.78 \\
\hline \multirow{5}{*}{$\begin{array}{l}\text { ICT use for } \\
\text { preparing } \\
\text { digital } \\
\text { educational } \\
\text { materials }\end{array}$} & College/BA/BSC & 1570.92 & \multirow[t]{5}{*}{88.385} & \multirow[t]{5}{*}{$<0.000$} & 3.80 & 3.85 \\
\hline & University/MA/MSC & 1869.63 & & & 4.25 & 4.42 \\
\hline & $\mathrm{PhD}$ & 2053.48 & & & 4.54 & 4.68 \\
\hline & $\begin{array}{l}\text { Vocational education } \\
\text { teacher }\end{array}$ & 931.50 & & & 3.00 & 3.00 \\
\hline & $\begin{array}{l}\text { secondary } \\
\text { education/maturation/NTR }\end{array}$ & 2111.68 & & & 4.60 & 5.00 \\
\hline \multirow{5}{*}{$\begin{array}{l}\text { Supporting the } \\
\text { learning } \\
\text { process through } \\
\text { digital } \\
\text { communication } \\
\text { options }\end{array}$} & College/BA/BSC & 1626.47 & \multirow[t]{5}{*}{38.465} & \multirow[t]{5}{*}{$<0.000$} & 4.53 & 4.74 \\
\hline & University/MA/MSC & 1823.11 & & & 4.80 & 5.07 \\
\hline & $\mathrm{PhD}$ & 1878.85 & & & 4.88 & 5.13 \\
\hline & $\begin{array}{l}\text { Vocational education } \\
\text { teacher }\end{array}$ & 1133.25 & & & 4.00 & 4.00 \\
\hline & $\begin{array}{l}\text { secondary } \\
\text { education/maturation/NTR }\end{array}$ & 1968.83 & & & 5.00 & 5.21 \\
\hline \multirow{5}{*}{$\begin{array}{l}\text { ICT use for } \\
\text { monitoring and } \\
\text { evaluation }\end{array}$} & College/BA/BSC & 1621.04 & \multirow[t]{5}{*}{44.120} & \multirow[t]{5}{*}{$<0.000$} & 3.76 & 3.84 \\
\hline & University/MA/MSC & 1821.56 & & & 4.07 & 4.27 \\
\hline & $\mathrm{PhD}$ & 2042.24 & & & 4.40 & 4.71 \\
\hline & education & 1348.25 & & & 3.50 & 3.50 \\
\hline & $\begin{array}{l}\text { secondary } \\
\text { education/maturation/NTR }\end{array}$ & 2139.48 & & & 4.55 & 4.80 \\
\hline \multirow{5}{*}{$\begin{array}{l}\text { Simultaneous } \\
\text { use of several } \\
\text { ICT services }\end{array}$} & College/BA/BSC & 1556.15 & \multirow[t]{5}{*}{108.887} & \multirow[t]{5}{*}{$<0.000$} & 3.82 & 3.97 \\
\hline & University/MA/MSC & 1880.37 & & & 4.34 & 4.64 \\
\hline & $\mathrm{PhD}$ & 2257.26 & & & 4.92 & 5.26 \\
\hline & $\begin{array}{l}\text { Vocational } \\
\text { teacher }\end{array}$ & 935.50 & & & 3.00 & 3.00 \\
\hline & $\begin{array}{l}\text { secondary } \\
\text { education/maturation/NTR }\end{array}$ & 1907.43 & & & 4.35 & 4.75 \\
\hline \multirow{5}{*}{$\begin{array}{l}\text { Only } \\
\text { reliable } \\
\text { systems }\end{array}$} & College/BA/BSC & 1689.89 & \multirow[t]{5}{*}{10.024} & \multirow[t]{5}{*}{0.040} & 4.81 & 5.12 \\
\hline & University/MA/MSC & 1761.13 & & & 4.93 & 5.19 \\
\hline & $\mathrm{PhD}$ & 1965.36 & & & 5.19 & 5.39 \\
\hline & $\begin{array}{l}\text { Vocational } \\
\text { teacher }\end{array}$ & 956.25 & & & 4.00 & 4.00 \\
\hline & $\begin{array}{l}\text { secondary } \\
\text { education/maturation/NTR }\end{array}$ & 1933.88 & & & 5.05 & 5.40 \\
\hline
\end{tabular}


Table 12

ICT use in light of the highest level of education by Slovakian pedagogues

\begin{tabular}{|c|c|c|c|c|c|c|}
\hline \multicolumn{7}{|c|}{ Slovakian- Kruskal-Wallis-probe: Correlation between highest qualification and teachers' ICT use } \\
\hline $\begin{array}{l}\text { Dependent } \\
\text { variable }\end{array}$ & Independent variable: & MR & $\begin{array}{l}\text { Chi- } \\
\text { square }\end{array}$ & $\mathrm{p}$ & average & $\begin{array}{l}\text { group } \\
\text { median }\end{array}$ \\
\hline \multirow{3}{*}{$\begin{array}{l}\text { Possession of } \\
\text { basic ICT skills }\end{array}$} & College/BA/BSC & 124.88 & \multirow[t]{3}{*}{1.947475} & \multirow[t]{3}{*}{0.378} & 5.50 & 5.50 \\
\hline & University/MA/MSC & 95.55 & & & 4.83 & 5.14 \\
\hline & PhD (doctorate) & 110.32 & & & 5.09 & 5.44 \\
\hline \multirow{3}{*}{$\begin{array}{l}\text { Confident } \\
\text { application of } \\
\text { ICT in teaching }\end{array}$} & College/BA/BSC & 76.13 & \multirow[t]{3}{*}{0.636918} & \multirow[t]{3}{*}{0.727} & 4.50 & 4.50 \\
\hline & University/MA/MSC & 97.36 & & & 4.88 & 5.12 \\
\hline & $\mathrm{PhD}$ (doctorate) & 98.77 & & & 4.82 & 5.14 \\
\hline \multirow{3}{*}{$\begin{array}{l}\text { ICT use in } \\
\text { preparing digital } \\
\text { education } \\
\text { materials }\end{array}$} & College/BA/BSC & 85.00 & \multirow[t]{3}{*}{0.521178} & \multirow[t]{3}{*}{0.771} & 4.50 & 4.50 \\
\hline & University/MA/MSC & 96.71 & & & 4.68 & 4.93 \\
\hline & $\mathrm{PhD}$ (doctorate) & 106.14 & & & 4.73 & 5.25 \\
\hline \multirow{3}{*}{$\begin{array}{l}\text { Supporting the } \\
\text { learning process } \\
\text { through digital } \\
\text { communication } \\
\text { options }\end{array}$} & College/BA/BSC & 114.38 & \multirow[t]{3}{*}{1.377140} & \multirow[t]{3}{*}{0.502} & 5.25 & 5.25 \\
\hline & University/MA/MSC & 95.73 & & & 5.03 & 5.28 \\
\hline & $\mathrm{PhD}$ (doctorate) & 111.23 & & & 5.00 & 5.33 \\
\hline \multirow{3}{*}{$\begin{array}{lr}\text { ICT use for } \\
\text { monitoring and } \\
\text { evaluation }\end{array}$} & College/BA/BSC & 107.63 & \multirow[t]{3}{*}{0.470725} & \multirow[t]{3}{*}{0.790} & 4.75 & 4.97 \\
\hline & University/MA/MSC & 97.33 & & & 4.44 & 4.70 \\
\hline & $\mathrm{PhD}$ (doctorate) & 87.86 & & & 4.18 & 4.50 \\
\hline \multirow{3}{*}{$\begin{array}{l}\text { Simultaneous } \\
\text { use of several } \\
\text { ICT services }\end{array}$} & College/BA/BSC & 94.75 & \multirow[t]{3}{*}{0.421697} & \multirow[t]{3}{*}{0.810} & 4.25 & 5.00 \\
\hline & University/MA/MSC & 96.42 & & & 4.53 & 4.74 \\
\hline & PhD (doctorate) & 107.23 & & & 4.82 & 5.13 \\
\hline \multirow{3}{*}{$\begin{array}{l}\text { Only uses } \\
\text { reliable systems }\end{array}$} & College/BA/BSC & 133.13 & \multirow[t]{3}{*}{3.382616} & \multirow[t]{3}{*}{0.184} & 5.75 & 5.75 \\
\hline & University/MA/MSC & 97.38 & & & 4.989 & 5.234 \\
\hline & PhD (doctorate) & 77.68 & & & 4.545 & 4.80 \\
\hline
\end{tabular}

Table 13

ICT use in light of the highest level of education by Serbian pedagogues

\begin{tabular}{|c|c|c|c|c|c|c|}
\hline $\begin{array}{l}\text { Dependent } \\
\text { variable }\end{array}$ & $\begin{array}{l}\text { Independent variable: } \\
\text { Highest qualification }\end{array}$ & $\mathrm{MR}$ & $\begin{array}{l}\text { Chi- } \\
\text { square }\end{array}$ & $\mathrm{p}$ & average & $\begin{array}{l}\text { group } \\
\text { median }\end{array}$ \\
\hline \multirow{3}{*}{$\begin{array}{l}\text { Possession of } \\
\text { basic ICT skills }\end{array}$} & College/BA/BSC & 52.00 & \multirow[t]{3}{*}{1.635904} & \multirow[t]{3}{*}{0.441} & 4.00 & 4.00 \\
\hline & University/MA/MSC & 70.07 & & & 5.21 & 5.53 \\
\hline & $\mathrm{PhD}$ (doctorate) & 75.38 & & & 5.48 & 5.64 \\
\hline \multirow{3}{*}{$\begin{array}{l}\text { Confident } \\
\text { application of } \\
\text { ICT in teaching }\end{array}$} & College/BA/BSC & 78.75 & \multirow[t]{3}{*}{1.240407} & \multirow[t]{3}{*}{0.538} & 5.00 & 5.00 \\
\hline & University/MA/MSC & 68.72 & & & 5.03 & 5.31 \\
\hline & PhD (doctorate) & 77.10 & & & 5.28 & 5.25 \\
\hline \multirow{3}{*}{$\begin{array}{l}\text { ICT use for } \\
\text { preparing digital } \\
\text { educational } \\
\text { materials }\end{array}$} & College/BA/BSC & 78.00 & \multirow[t]{3}{*}{0.359129} & \multirow[t]{3}{*}{0.836} & 5.25 & 5.25 \\
\hline & University/MA/MSC & 69.65 & & & 5.10 & 5.41 \\
\hline & PhD (doctorate) & 73.06 & & & 5.16 & 5.52 \\
\hline \multirow{3}{*}{$\begin{array}{l}\text { Supporting the } \\
\text { learning process } \\
\text { through digital } \\
\text { communication } \\
\text { options }\end{array}$} & College/BA/BSC & 75.38 & \multirow[t]{3}{*}{0.536582} & \multirow[t]{3}{*}{0.765} & 5.00 & 5.00 \\
\hline & University/MA/MSC & 71.35 & & & 5.24 & 5.51 \\
\hline & PhD (doctorate) & 65.96 & & & 5.20 & 5.41 \\
\hline \multirow{3}{*}{$\begin{array}{lr}\text { ICT use for } \\
\text { monitoring and } \\
\text { evaluation }\end{array}$} & College/BA/BSC & 88.25 & \multirow[t]{3}{*}{0.969223} & \multirow[t]{3}{*}{0.616} & 5.50 & 5.50 \\
\hline & University/MA/MSC & 69.59 & & & 4.88 & 5.24 \\
\hline & $\mathrm{PhD}$ (doctorate) & 71.72 & & & 4.92 & 5.35 \\
\hline
\end{tabular}




\begin{tabular}{|c|c|c|c|c|c|c|}
\hline \multirow{3}{*}{$\begin{array}{l}\text { Simultaneous use } \\
\text { of several ICT } \\
\text { services }\end{array}$} & College/BA/BSC & 79.00 & \multirow[t]{3}{*}{0.591387} & \multirow[t]{3}{*}{0.744} & 5.00 & 5.00 \\
\hline & University/MA/MSC & 69.34 & & & 4.86 & 5.31 \\
\hline & $\mathrm{PhD}$ (doctorate) & 74.30 & & & 5.00 & 5.50 \\
\hline \multirow{3}{*}{$\begin{array}{l}\text { Only uses } \\
\text { reliable systems }\end{array}$} & College/BA/BSC & 55.25 & \multirow[t]{3}{*}{0.727308} & \multirow[t]{3}{*}{0.695} & 4.75 & 4.67 \\
\hline & University/MA/MSC & 70.58 & & & 4.95 & 5.30 \\
\hline & $\mathrm{PhD}$ (doctorate) & 72.58 & & & 5.08 & 5.35 \\
\hline
\end{tabular}

The Kruskal-Wallis probe was applied to identify whether the highest level of qualification has any impact on ICT use. The analysis of Hungarian respondents' answers reveals a pattern according to which the higher the level of qualification, the better the given educator considers their own habits, skills, and efficiency of ICT use. In case of all dependent variables significant divergences were discerned. Responses from Slovakia and Serbia do not show such uniformity as no significant divergence can be discerned in the samples. Thus, we can conclude that ICT use is not dependent on educational qualification and the high $\mathrm{p}$ values suggest that any differences in the answers are merely coincidental.

\section{Conclusions and Future Work}

In March 2020 it was revealed that the Hungarian education system was not prepared to meet the requirements of $21^{\text {st }}$ Century education. Teachers had merely, one weekend, to change from the delivery based, frontal instruction and monitoring paradigm, to become inclusive and adaptive organisers of the learning process. Educators were also expected to promote student motivation in a playful and enjoyable manner. The real division between the two approaches, was not familarity with, or previous user experience, concerning digital devices, or the possession of digital literacy, but, the maintenance of a flexible pedagogical perspective. The substantial challenge imposed by the digital transformation process, was convoyed with several potential setbacks, including the limited control of student activities, via the digital surfaces and the reduced attention span of students, despite the application of innovative and interactive solutions. While previous experiences gained from distance learning provided an adequate foundation for achieving the respective goals, the VUCA effect imposed heavy demand on everyone. The present research confirmed that, apart from using such platforms, such as, the Edupage or Big Blue Button, the digital device system of the three countries under inquiry is virtually identical. The ICT habits and device use perceived in case of the large sample groups showed a wide variety of solutions depending on the available digital skills and infrastructure. The examination of numerous background variables revealed significant divergence only in two aspects: Confident ICT use and the preparation of digital educational materials. Although participation in digital training programs along with the educational level can have a major impact, in light of the $\mathrm{p}<0.05$ reliability, significant divergences were not revealed.

Modern education methods prioritise fast identification and retrieval of authentic and credible information, over passive reception of knowledge, while problem solving, replaces following the teacher's instructions. Proven by the results of 
final examinations administered on-line, such skills and aptitudes have to be continuously developed. In addition, Educators must pay attention to such concerns, in the future.

\section{References}

[1] B. Orosz, et. al.: Digital education in digital cooperative environments. Journal of Applied Technical and Educational Sciences, Vol. 9, No. 4, 2019, pp. 55-69

[2] A. Buda: Educators in the digital age. Trend research in schools in a big city. Budapest, Hungary: Thought Publisher 2020

[3] B. Csapó: The role of teacher knowledge in the development of the education system, NEW PEDAGOGICAL REVIEW, Vol 3, No. 4, 2007, pp. 1-9

[4] T. Sebestyén: (2005): Human capital and education. Which quality do we measure? Labor Review, april, 2005, pp 28-32

[5] Eric A. Hanushek, Dennis D. Kimko: Schooling, Labor-Force Quality, and the Growth of Nations, American Economic Review, Vol. 90, No. 5, 2000, pp. 1184-1208

[6] Rivkin, S. G., E. A. Hanushek, J. F. Kain: Teachers, schools, and academic achievement, Econometrica, 2005

[7] Nagy et al.: Development opportunities of digital and labor market competences in higher education, In: L., Gómez Chova; A., López Martínez; I., Candel Torres (ed.) ICERI2018 Proceedings: $11^{\text {th }}$ International Conference of Education, Research and Innovation, Seville, International Academy of Technology, Education and Development (IATED), 201810 600 p. pp. $10590-10594$

[8] A. Kovari: Synergy of digital society and digital education. Civil Szemle, Vol. 17, No. 1, 2020, pp. 69-72

[9] A. Kovari, J. Katona, C. Costescu: Quantitative Analysis of Relationship Between Visual Attention and Eye-Hand Coordination. Acta Polytechnica Hungarica, Vol. 17, No. 2, 2020, pp 77-95

[10] L. Besenyei: The revolution of generation change, OPUS ET EDUCATIO, Vol. 3, No. 4, doi:http://dx.doi.org/10.3311/ope.19, 2016, pp. 371-378

[11] P. Molnár: Networking and learning in network environments, Budapest, Eötvös Loránd University, 2013, http://www.tankonyvtar.hu/hu/tartalom/tamop412A/20110073_halozatosodas_tanulas_halozati_kornyezetben/index.html

[12] L. Z. Karvalics: Information, society, history, Budapest, Typotex, 2003, $178 \mathrm{p}$. 
[13] G. Siemens: Connectivism: A Learning Theory for the Digital Age. 2 International Journal of Instructional Technology and Distance Learning, 2004, http://www.itdl.org/journal/jan_05/article01.htm

[14] S. Forgó: New Media competence perspectives - instructor competences required for teaching the New Media, In Nádasi András (ed.): Agria Média 2011 - X. Information Technology and Educational Technology Conference and Exhibition. Eger, Líceum, 2011, pp. 216-217

[15] P. Baranyi, A. Csapo, Gy. Sallai: Cognitive Infocommunications (CogInfoCom), Springer International Publishing Switzerland, 2015, p. 219

[16] P. Baranyi, A. Csapó: Definition and synergies of cognitive infocommunications. Acta Polytechnica Hungarica, Vol. 9, No. 1, 2012, pp. $67-83$

[17] A. Kovari, M. Rajcsányi-Molnár: Mathability and Creative Problem Solving in the MaTech Math Competition. Acta Polytechnica Hungarica, Vol. 17, No. 2, 2020, pp. 147-161

[18] E. Kocsó, M. Cserné Pekkel, L. Bognár, P. Horváth: Teaching Mathematics at the Correspondence Courses of the University of Dunaújváros. Journal of Applied Technical and Educational Sciences, Vol. 10, No. 4, 2020, pp 87104

[19] A. Skobrák: Direct hand-movement control in virtual space: a potential interface for virtual lab purposes. Transactions on IT and Engineering Education, Vol. 2, No. 1, 2019, pp 30-45

[20] G. Sziládi G.: Applications of human-computer interfaces and related psychological-educational aspects. Computers \& Learning, Vol. 2, No. 1, 2019, pp. 1-14

[21] R. Demeter et al: Importance of digital simulation in the competence development of engineers defining the society of the future, Civil Szemle, Vol. 17, No. 2, 2020, pp. 89-101

[22] C. Costescu, A. M. Rosan: Development an assessment protocol to identify the characteristics of ASD using eye-tracking for special education purpose. Journal of Applied Technical and Educational Sciences, Vol. 9, No. 4, 2019, pp. 70-87

[23] S. Maravic Cisar, R. Pinter: Analysis of students' dropout rate at Subotica Tech. Journal of Applied Technical and Educational Sciences, Vol. 9, No. 4, 2019, pp. 43-54

[24] A. Kovari: Study of Algorithmic Problem-Solving and Executive Function. Acta Polytechnica Hungarica, Vol. 17, No. 9, 2020, pp. 241-256 